\title{
COMUNicAÇÃo breve Adaptação da Escala de Silhuetas Brasileiras para uso digital
}

\author{
Adapting Brazilian Silhouette Scale for digital use \\ Simone Cardoso Freire' ${ }^{1}$ Mauro Fisberg²
}

\section{Palavras-chave}

Imagem corporal, adulto jovem, digital, reprodutibilidade de testes, escalas.

\section{Keywords}

Body image, young adult, digital, test reproducibility, scales.

\section{RESUMO}

Objetivo: Adaptar a escala de silhuetas originais em cartões de papel e validar para uso digital. Métodos: Participaram dessa pesquisa 88 jovens, os quais foram avaliados quanto ao índice de massa corporal (IMC) e questões sobre a imagem corporal com as escalas de silhuetas de papel e digital em relação ao corpo atual (IMC-A) e o corpo desejado (IMC-D). O processo de validação da escala digital se dá pela comparação entre o IMC com o IMC-A. Resultados: Os resultados foram satisfatórios $(0,70)$ para o grupo quando avaliado em conjunto e para o sexo masculino. No entanto, para o sexo feminino obtivemos um valor relativamente baixo, na concordância de Lin $(0,64)$, mas com valores de ICC $(0,89)$ e correlação de Spearman $(0,85)$ aceitáveis. Conclusão: As imagens no computador podem ser utilizadas para a avaliação da imagem corporal de jovens. Porém, é mais seguro realizar associações com questões referentes à prática de atividade física e do comportamento alimentar que, em conjunto, possam auxiliar na avaliação da insatisfação corporal.

\section{ABSTRACT}

Objective: Adapt The Brazilian Silhouette Scale, rendering them fit for digital use. Methods: Eighty-eight young people took part in the survey, in which they were assessed regarding their body mass index (BMI) and topics related to body image, with the use of paper and digital silhouettes in relation to the current body (BMI-C) and the desired body (BMI-D). The process used to validate the digital scale is done by comparing the BMI with BMI-C. Results: Were satisfactory (0.70) for the group, as a whole and for the males included. However, females showed a relatively low value at Lin's concordance (0.64), but with acceptable ICC (0.89) and Spearman (0.85) values. Conclusion: The computer images can be used to assess body image for young people. However, it is safer to make associations with topics regarding physical activity and eating behavior, which together can help us assess body dissatisfaction.

1 Universidade Federal de São Paulo, Escola Paulista de Medicina (Unifesp/EPM), Programa de Pós-Graduação em Ciências Aplicadas à Pediatria.

2 Universidade Federal de São Paulo, Escola Paulista de Medicina (Unifesp/EPM), Departamento de Pediatria e Programa de Pós-Graduação em Ciências Aplicadas à Pediatria. Hospital Infantil Sabará, Fundação José Luiz Setubal, Centro de Dificuldades Alimentares do Instituto Pensi. 


\section{INTRODUÇÃO}

A imagem corporal é a imagem que temos na mente do tamanho, aparência e forma do corpo assim como dos sentimentos a respeito dessas características e das partes que o formam. Há dois componentes que integram a construção da imagem corporal dada pelo aspecto cognitivo comportamental: a satisfação e a insatisfação'.

Há um aumento da utilização das escalas de imagem na literatura, pois é um dos meios para realizar a autoavaliação da imagem corporal. Elas foram desenvolvidas para avaliar indivíduos com distorções da imagem corporal que apresentassem distúrbios alimentares como a anorexia e a bulimia nervosa².

Esse tipo de instrumento vem sendo utilizado para avaliar diversos grupos etários ${ }^{3-5}$, em que geralmente as mulheres mais jovens vêm apresentando insatisfação corporal dada a influência dos padrões sociais, a necessidade de ser magro, principalmente pela influência da mídia, família e amigos ${ }^{3,5-7}$.

A insatisfação corporal está associada a mudanças no padrão comportamental tanto em relação à alimentação como à prática de atividades físicas, visto que ambos estão correlacionados. Por esse fato, a American Dietetic Association registrou em 2006 o termo disordered eating, ou comer transtornado, associado a práticas inadequadas para manter o peso baixo ou controlar o peso, com excesso de algum tipo de atividade física na maioria dos casos, os quais são considerados comportamentos de risco para os transtornos alimentares ${ }^{6}$.

A utilização das escalas de silhuetas é única para cada população e faixa etária, assim como do seu formato original. Na alteração dessas condições, deve-se realizar o processo de adaptação para, então, verificar sua validade. Tem-se observado muitos trabalhos de adaptações de escalas de silhuetas pelo aumento dos estudos referentes à imagem corporal e, consequentemente, seu uso na prática clínica. O instrumento permite que o indivíduo realize a autoavaliação tanto no que se refere ao corpo atual quanto ao corpo desejado, pois a partir dessa diferença obtém-se a insatisfação corpora|3,7-9.

A escala de silhuetas brasileiras foi desenvolvida para utilização com cartões de papel, e o nosso trabalho foi realizar a adaptação e validação para uso digital com a finalidade de viabilizar o processo de avaliação com o uso de programas computadorizados, aumentando e facilitando a aplicação de questionários online que possam colaborar com pesquisas e contribuir com medidas preventivas para a diminuição dos comportamentos de riscos para os transtornos alimentares.

\section{MÉTODOS}

Para a adaptação da escala de imagem digital foram selecionados somente jovens universitários de 18 a 25 anos, estudantes regularmente matriculados nos cursos de filosofia, história, história da arte, letras e pedagogia do campus Gua- rulhos da Universidade Federal de São Paulo (Unifesp), no período de maio a junho de 2013.

Foram excluídos alunos com vínculo empregatício ou estudantil na área da saúde, com o objetivo de não apresentar viés nas respostas referentes à imagem corporal, pois, no trabalho sequencial do uso do questionário sobre saúde, alimentação e percepção corporal, esses critérios também fizeram parte do grupo de exclusão.

O número de participantes para a adaptação da escala de silhuetas foi definido com base na elaboração do artigo original ${ }^{7}$, utilizando como parâmetros o poder de 95\% e nível de significância de 5\%, encontrando-se o valor de 70 indivíduos.

Os alunos foram pesados e medidos com balança mecânica da marca Welmy, com capacidade para 150 kg, graduação de 100 gramas e estadiômetro. Os valores de peso e estatura foram utilizados para estabelecer o índice de massa corporal (IMC) - medida do peso corporal expresso em kg dividido pela estatura em $\mathrm{m}^{2}$, denominada neste trabalho como IMC Real (IMC-R), de acordo com a classificação da Organização Mundial da Saúde ${ }^{10}$.

Depois, os participantes foram questionados quanto à percepção corporal nos dois tipos de escalas de silhuetas: em cartões de papel ${ }^{7}$ e na tela do computador, com as mesmas imagens, alterando somente o tamanho e o tipo de visualização. As duas etapas foram realizadas na sequência no Núcleo de Apoio ao Estudante sem tempo de intervalo, como alguns autores recomendam ${ }^{4,5,7}$.

Os estudantes observaram primeiro a escala de papel e depois a escala digital e responderam a duas questões em cada etapa:

1. Aponte a figura que melhor represente o seu corpo atualmente, a qual foi determinada nesse trabalho de IMC Atual (IMC-A).

2. Aponte a figura que melhor represente o corpo que gostaria de ter, a qual foi determinada nesse trabaIho de IMC Desejado (IMC-D).

A escala utilizada na forma original de Kakeshita et al. ${ }^{7}$ é composta de 15 figuras em cartões plastificados de $6,5 \mathrm{~cm}$ de largura por 12,5 cm de altura, com a figura centralizada em fundo preto, contornada por margens a $0,5 \mathrm{~cm}$ equidistantes das bordas da figura e do cartão, com as 15 figuras dispostas horizontalmente. Para obter a mesma imagem e a mesma técnica no processo de digitalização, haveria diminuição significativa das figuras. Por isso, optou-se por organizar as figuras em duas fileiras, sendo a primeira com oito figuras dentre os IMCs de $12,5 \mathrm{~kg} / \mathrm{m}^{2}$ a $30 \mathrm{~kg} / \mathrm{m}^{2}$ e, na linha de baixo, sete figuras dentre os IMCs $32,5 \mathrm{~kg} / \mathrm{m}^{2}$ a $47,5 \mathrm{~kg} / \mathrm{m}^{2}$.

O tamanho das escalas digitalizadas para a adaptação foi de 480 × 340 pixels, sendo distribuídas em duas fileiras. A escala digital foi apresentada no software Microsoft Office PowerPoint 2007 no modo apresentação de slides, com uso de um notebook com tela de 16 polegadas. 
A adaptação da escala de imagem é parte da tese de doutorado intitulada de "Comportamento alimentar de jovens e sua relação com a comunicação social e de especialistas da saúde". Essa pesquisa teve aprovação do Comitê de Ética em Pesquisa da Universidade Federal de São Paulo - Escola Paulista de Medicina (Unifesp) pelo número 1.636/11 e está de acordo com os requisitos da resolução n 196/96 do Conselho Nacional de Saúde do Ministério da Saúde.

\section{Análise estatística}

Para avaliar a validade concorrente da escala de silhuetas digitais (ESD) em relação à escala de papel (ESP), foi utilizado o teste de correlação intraclasse (ICC), o qual aponta a confiabilidade entre as medidas nas duas escalas; a concordância de Lin, que analisa a reprodução dos instrumentos, e a correlação de Spearman (r), que aponta a correlação entre postos e linear entre as duas escalas.

Todos os testes foram realizados com os valores dos IMC-R, IMC-A e IMC-D das ESP e ESD. Todas as análises foram realizadas utilizando os softwares SPSS 17, Minitab 16 e Microsoft Office Excel 2007. Foi adotado nível de significância de $5 \%$ para todas as análises.

\section{RESULTADOS}

A pesquisa contou com a participação de 88 universitários, sendo 29 do sexo masculino e 59 do sexo feminino. Na Tabela 1, estão descritos os valores de idade, peso, estatura, IMC-R, IMC-A e IMC-D das ESP e ESD.

A Tabela 2 aponta os resultados dos testes de ICC, concordância de Lin e correlações de Spearman entre as ESP e ESD. Os testes foram realizados com a amostra total e separados por sexo com os IMC-R, IMC-A e IMC-D.

Os resultados do ICC foram $(\geq 0,70)$ para todo o grupo e também quando avaliados separado por sexo, sendo considerados satisfatórios ${ }^{11}$.

A concordância de Lin mostrou-se baixa para o sexo feminino na avaliação do IMC-R com o IMC-A da $\operatorname{ESP}(0,68)$ e da $\operatorname{ESD}(0,64)$, porém a concordância aumenta na avaliação do IMC-A e IMC-D para esse mesmo sexo. Para o sexo masculino, o IMC-D apresenta apenas o ICC satisfatório $(0,81)$, sendo as medidas de concordância de Lin $(0,65)$ e correlação de Spearman $(0,58)$ consideradas insatisfatórias.

A correlação de Spearman aponta valores satisfatórios em relação aos IMC-R e IMC-A das ESP e ESD. No entanto, ao

Tabela 1. Idade, peso, estatura, IMC-R, IMC-A e IMC-D das ESP e ESD, dos universitários da Unifesp, segundo sexo. Guarulhos, SP, Brasil, 2013

\begin{tabular}{|c|c|c|c|c|c|c|c|c|c|c|c|c|c|}
\hline \multirow[t]{2}{*}{ Variáveis } & \multicolumn{4}{|c|}{$\begin{array}{c}\text { Total } \\
(\mathrm{n}=88)\end{array}$} & \multicolumn{4}{|c|}{$\begin{array}{l}\text { Masculino } \\
(n=29)\end{array}$} & \multicolumn{4}{|c|}{$\begin{array}{l}\text { Feminino } \\
(\mathrm{n}=59)\end{array}$} & \multirow[t]{2}{*}{ P-valor } \\
\hline & Min & Máx & $\bar{x}(\mathrm{dp})$ & Md & Min & Máx & $\bar{x}(\mathrm{dp})$ & Md & $\operatorname{Min}$ & Máx & $\chi(d p)$ & Md & \\
\hline Idade (anos) & 18 & 25 & $21 \pm 2$ & 20 & 18 & 25 & $21 \pm 2$ & 21 & 18 & 25 & $20 \pm 2$ & 20 & 0,362 \\
\hline Peso (kg) & 44 & 152 & $67 \pm 18$ & 67 & 44 & 152 & $76 \pm 23$ & 72 & 44 & 106 & $63 \pm 14$ & 62 & 0,002 \\
\hline Estatura (cm) & 150 & 185 & $165 \pm 9$ & 168 & 162 & 185 & $175 \pm 6$ & 176 & 150 & 172 & $161 \pm 5$ & 160 & $<0,001$ \\
\hline IMC-R $\left(\mathrm{kg} / \mathrm{m}^{2}\right)$ & 16 & 44 & $25 \pm 6$ & 23 & 16 & 44 & $24 \pm 6$ & 23 & 16 & 40 & $25 \pm 5$ & 24 & 0,966 \\
\hline IMC-A (ESP) & 12,5 & 45 & $27 \pm 8$ & 26 & 12,5 & 45 & $25 \pm 9$ & 22 & 15 & 45 & $28 \pm 8$ & 30 & 0,042 \\
\hline IMC-A (ESD) & 12,5 & 45 & $28 \pm 7$ & 26 & 12,5 & 45 & $26 \pm 8$ & 22 & 15 & 45 & $29 \pm 7$ & 30 & 0,060 \\
\hline IMC-D (ESP) & 15 & 40 & $25 \pm 5$ & 26 & 15 & 40 & $26 \pm 5$ & 27 & 15 & 35 & $25 \pm 4$ & 25 & 0,509 \\
\hline IMC-D (ESD) & 15 & 40 & $26 \pm 5$ & 26 & 15 & 40 & $24 \pm 7$ & 25 & 17,5 & 40 & $27 \pm 4$ & 27 & 0,047 \\
\hline
\end{tabular}

IMC-R: índice de massa corporal real; IMC-A: índice de massa corporal atual; IMC-D: índice de massa corporal desejado; ESP: Escala de Silhuetas de Papel; ESD: Escala de Silhuetas Digital.

Tabela 2. Correlação intraclasse (ICC), concordância de Lin (Rho) e correlação de Spearman (r) entre as ESP e ESD. Guarulhos, SP, Brasil, 2013

\begin{tabular}{|c|c|c|c|c|c|c|c|c|c|}
\hline \multirow{2}{*}{ Variáveis } & \multicolumn{3}{|c|}{$\begin{array}{c}\text { Total } \\
(n=88)\end{array}$} & \multicolumn{3}{|c|}{$\begin{array}{c}\text { Masculino } \\
(n=29)\end{array}$} & \multicolumn{3}{|c|}{$\begin{array}{c}\text { Feminino } \\
(n=59)\end{array}$} \\
\hline & ICC & Rho Lin & $r$ & ICC & Rho Lin & $r$ & ICC & Rho Lin & $r$ \\
\hline IMC-RxIMC-A (ESP) & 0,90 & 0,76 & 0,88 & 0,94 & 0,88 & 0,92 & 0,89 & 0,68 & 0,89 \\
\hline IMC-RxIMC-A (ESD) & 0,89 & 0,73 & 0,84 & 0,93 & 0,87 & 0,85 & 0,89 & 0,64 & 0,85 \\
\hline IMC-A(ESP) x IMC-A (ESD) & 0,97 & 0,94 & 0,94 & 0,97 & 0,93 & 0,89 & 0,97 & 0,94 & 0,95 \\
\hline IMC-D(ESP) x IMC-D (ESD) & 0,85 & 0,73 & 0,72 & 0,81 & 0,65 & $0,58^{*}$ & 0,92 & 0,82 & 0,85 \\
\hline
\end{tabular}

Todos os valores- $p \leq 0,001$, exceto * $p 0,001$.

ICC: correlação intraclasse; Rho Lin: concordância de Lin; $r$ : correlação de Spearman.

IMC-R: índice de massa corporal real; IMC-A: índice de massa corporal atual; IMC-D: índice de massa corporal desejado; ESP: Escala de Silhuetas de Papel; ESD: Escala de Silhuetas Digital. 
avaliar o IMC-D para o sexo masculino, observamos o valor de $0,58(p=0,001)$, mostrando uma maior diferença em relação aos demais resultados.

\section{DISCUSSÃO}

Com o aumento da insatisfação corporal, os estudos de adaptação e validação de escalas de silhuetas têm sido evidenciados na literatura, pois o uso dessa ferramenta permite avaliar a percepção e a insatisfação corporal ${ }^{7,9}$.

De acordo com Stunkard'12, a variável de maior relevância é a correlação entre o IMC-R com o IMC-A da escala que foi adaptada, em que Thompson" sugere valores próximos a 0,70 como aceitável para o processo de validação. Nesse sentido, os resultados foram satisfatórios para ESD para todo o grupo. No entanto, para o sexo feminino a concordância de Lin apresentou 0,64 na ESD e 0,68 na ESP, ambos valores < 0,70.

Porém, os demais resultados das análises para o sexo feminino em relação à ESD foram satisfatórios, sendo a confiabilidade de 0,89 dada pelo ICC e a correlação de Spearman de 0,85 . Esses resultados mostram a possibilidade da utilização da ESD pelos valores de associação entre o IMC-R e o IMC-A.

Thompson ${ }^{11}$ acredita que o método estatístico não define a utilização das escalas de silhuetas. Alguns trabalhos vêm refinando as medidas psicométricas, realizando mais de um tipo de análise estatística para a complementação da hipótese para sua utilização, como foi realizado neste trabalho.

Sands et al..$^{13}$ validaram um programa computadorizado com fotografias de tamanhos de $400 \times 160$ pixels com somente três imagens: atual, diminuída e aumentada, comparando com o uso da escala de silhuetas de Stunkard ${ }^{12}$, relacionando com partes do corpo. A escala computadorizada mostrou ser mais efetiva para se correlacionar com partes do corpo quando se refere à insatisfação corporal em mulheres jovens. Isso se deu pelo refinamento das fotografias em comparação às escalas que mantêm estilizado e anônimo o seu formato, demonstrando que o uso do computador e a diminuição das figuras não alteram o resultado final, mas neste caso são mais reais quanto ao formato do contorno corporal ${ }^{13}$.

Especialistas apontam para o cuidado na avaliação da imagem corporal para o atual momento da relação do culto ao corpo, em que mulheres querem ser cada vez mais magras $^{2,3,6,7,9}$, e homens, mais musculosos ${ }^{14}$. A literatura mostra que há prática do exercício excessivo para homens $s^{6,14}$ e hábitos não saudáveis para as mulheres $2,3,9,1,14$, e ambos correlacionam com a subjetividade da percepção corporal tanto para menos como para mais ${ }^{12,15}$.

Pesquisadores relatam que para esse perfil de homens há dificuldade em se adaptar às avaliações que utilizam as figuras das escalas de silhuetas tradicionais, podendo ser mais um dos fatores para a baixa correlação entre as variáveis analisadas ${ }^{9,14,15}$.
Podemos observar essa ocorrência nos resultados do IMC-D, os quais nesse trabalho foram baixos para o sexo masculino na concordância de Lin $(0,65)$ e na correlação de Spearman $(0,58)$. Alguns autores apontam que uma menor correlação na avaliação do IMC-D para homens pode sugerir a não importância dada ao corpo, enquanto o sexo feminino mostra uma maior preocupação $0^{5,79}$. Esses dados mostram a interação com o aumento da insatisfação corporal por jovens com peso adequado e não somente por indivíduos obesos ${ }^{7}$, ou com presença de distúrbios alimentares ${ }^{3,6}$.

Nesse trabalho não foram realizados os testes por subgrupos referentes à classificação do estado nutricional e de insatisfação corporal. Ambos podem contribuir na compreensão dos casos com menor concordância ou correlação entre as variáveis avaliadas, sendo explicadas pela diferença da massa corporal ou pela insatisfação corporal ${ }^{7}$.

\section{CONCLUSÃO}

Podemos utilizar as escalas de silhuetas brasileiras digitalizadas como forma de avaliação da percepção e insatisfação corporal em jovens brasileiros, permitindo avaliar a imagem corporal. A reprodutibilidade da escala digital contribui de forma efetiva na construção de questionários on line para a avaliação dos comportamentos de riscos para os transtornos alimentares.

\section{CONTRIBUIÇÕES INDIVIDUAIS}

Simone Cardoso Freire - Formulou o desenho do estudo, realizou a coleta, analisou e interpretou os dados e escreveu o artigo.

Mauro Fisberg - Participou da formulação do desenho do estudo, interpretou os dados e realizou criticamente a revisão final do manuscrito.

\section{CONFLITOS DE INTERESSE}

Não há conflitos de interesse a serem declarados.

\section{AGRADECIMENTOS}

Eu, Simone Cardoso Freire, nutricionista, aluna do Programa de Pós-graduação em Ciências Aplicadas à Pediatria da Unifesp, e Mauro Fisberg, médico, orientador do Programa de Pós-graduação em Ciências Aplicadas à Pediatria da Unifesp, agradecemos a Coordenação de Aperfeiçoamento de Nível Superior (Capes) pela bolsa concedida. 


\section{REFERÊNCIAS}

1. Cash TF. Cognitive-Behavior Perspective on Body Image. In: Cash TF, Pruzinsky T. Body image - A handbook of theory, research, \& clinical practice. New York: The Guilford Press; 2002. p. 38-46.

2. Thompson MA, Gray JJ. Development and validation of a new body-image assessment scale. J Pers Asses. 1995;64(2):258-69.

3. Neumark-Sztainer D, Falkner N, Story M, Perry C, Hannan PJ, Mulert S. Weight-teasing among adolescents: correlations with weight status and disordered eating behaviors. Int J Obes Relat Metab Disord. 2002;26(1):123-31.

4. Kakeshita IS, Almeida SS. Relação entre índice de massa corporal e a percepção da autoimagem em universitários. Rev Saude Publica. 2006;40(3):497-504.

5. Alvarenga MS, Philippi ST, Lourenço BH, Sato PM, Scagliusi FB. Insatisfação com a imagem corporal em universitárias brasileiras. J Bras Psiquiatr. 2010;59(1):44-51.

6. ADA Report. Position of the American Dietetic Association: Nutrition intervention in the treatment of anorexia nervosa, bulimia nervosa, and other eating disorders. J Am Diet Assoc. 2006;106(12):2073-82.

7. Kakeshita IS, Silva AIP, Zanatta DP, Almeida SS. Construção e fidedignidade teste-reteste de escalas de silhuetas brasileiras para adultos e crianças. Psic Teor Pesq. 2009;25(2):263-70.
8. Stunkard AJ, Sørensen T, Schulsinger F. Use of the Danish Adoption Register for the study of obesity and thinness. In: Kety SRL, Sidman R, Matthysse, Sanchez-Villegas, A, editor. The genetics of neurological and psychiatric disorders. New York: Raven Press; 1983. p. 115-20.

9. Williamson DA, Davis C, Bennett SM, Goreczny AJ, Gleaves DH. Development of a simple procedure for assessing body image disturbances. Behav Assess. 1989;11(4):433-46.

10. OWH. Obesity: preventing and managing the global epidemic: World Health Organization; 2000.

11. Thompson JK. The (mis)measurement of body image: ten strategies to improve assessment for applied and research purposes. Body Image. 2004;1(1):7-14.

12. Stunkard A. Old and new scales for the assessment of body image. Percept Mot Skills. 2000;90(3 Pt 1):930.

13. Sands R, Maschette W, Armatas C. Measurement of body image satisfaction using computer manipulation of a digital image. J Psychol. 2004;138(4):325-37.

14. Smolak L, Murnen SK, Thompson JK. Sociocultural influences and muscle building in adolescent boys. Psychol Men Masc. 2005;6(4):227-39.

15. Brdarić $D$, Jovanović , Gavrilov-Jerković $V$. The relationship between body mass index and subjective well-being-the moderating role of body dissatisfaction. Med Pregl. 2015;68(910):316-23. 